\title{
INFLUÊNCIA DA FISIOTERAPIA RESPIRATÓRIA NA PRESSÃO INTRACRANIANA EM PACIENTES COM TRAUMATISMO CRANIENCEFÁLICO GRAVE
}

\author{
Rosana A. Thiesen ${ }^{1}$, Desanka Dragosavac', Augusto C. Roquejani ${ }^{3}$, \\ Antônio L.E. Falcão ${ }^{4}$, Sebastião Araujo ${ }^{5}$, Venâncio P. Dantas Filho ${ }^{6}$, \\ Rosmari A.R.A. Oliveira7, Renato G.G. Terzi ${ }^{8}$
}

\begin{abstract}
RESUMO - Objetivo:Estudar a influência das manobras de fisioterapia respiratória na pressão intracraniana (PIC) dos pacientes com trauma craniencefálico grave. Método: Trinta e cinco pacientes com trauma craniencefálico grave foram incluídos no estudo, sendo divididos em três grupos: com PIC $\leq 10,11-20$ e 21-30 $\mathrm{mmHg}$. As variáveis monitorizadas foram: PIC e pressão arterial média. A pressão de perfusão cerebral foi calculada pela diferença de pressão arterial média e PIC. Resultados: A manobra de aspiração traqueal causou aumento de PIC em todos os grupos. A pressão arterial média não teve alterações e a pressão de perfusão cerebral diminuiu pouco, porém mantendo valores normais. Conclusão: As manobras de fisioterapia respiratória podem ser usadas com segurança em pacientes com traumatismo craniencefálico grave, com PIC abaixo de $30 \mathrm{mmHg}$.Certo cuidado deve ser tomado durante a aspiração traqueal.
\end{abstract}

PALAVRAS-CHAVE: traumatismo craniencefálico, fisioterapia respiratória, pressão intracraniana, pressão de perfusão cerebral.

\begin{abstract}
Influence of the respiratory physioterapy on intracranial pressure in severe head trauma patients
ABSTRACT - Objective: To evaluate influence of the respiratory physiotherapy on intracranial pressure (ICP) in patients with severe head trauma. Method: Thirty five patients with severe head trauma were included in the study.The patients were divided into three groups: ICP 0-10, 11-20 and 21-30 mmHg. The following variables were measured: ICP and mean arterial pressure. Cerebral perfusion pressure was calculated as the difference between mean arterial and intracranial pressure. Results. Endotracheal aspiration increased ICP in all patients. The mean arterial pressure didn't change and cerebral perfusion pressure decreased, but remaning normal value. Conclusion: Respiratory physiotherapy maneuvers can be safely applied in patients with severe head trauma with ICP below $30 \mathrm{mmHg}$. More attention should be taken during endotracheal aspiration .
\end{abstract}

KEY WORDS: head injury, respiratory physioterapy, intracranial pressure, cerebral perfusion pressure.

Os pacientes com traumatismo craniencefálico (TCE) grave, com escala de coma de Glasgow igual ou menor que 8 , são geralmente submetidos à monitorização da pressão intracraniana (PIC), e da pressão arterial média (PAM) e tratados de acordo com um protocolo rígido de assistência, que inclui sedação, elevação de cabeceira do leito, ventilação mecânica otimizada, fisioterapia respiratória e motora, entre outras ${ }^{1,2}$. A aplicação das manobras cinéticas de fisioterapia respiratória promove aumento momentâneo da pressão intratorácica, diminuindo o retorno venoso cerebral e elevando a PIC nesses $p a-$ cientes $^{3-6}$.

Sabe-se que as alterações da PIC influenciam diretamente a pressão de perfusão cerebral (PPC), que é calculada como diferença entre a PAM e a PIC. Portanto, aumentos da PIC poderiam acarretar diminuição da PPC, se não houver aumento conco-

\footnotetext{
Unidade de Terapia Intensiva do Hospital das Clinicas da Universidade Estadual de Campinas, UNICAMP; Departamento de Neurocirurgia do HC UNICAMP, Campinas SP, Brasil: ${ }^{1}$ Fisioterapeuta, Mestra, Professora da Universidade Paulista, Campus Campinas/Sorocaba; ${ }^{2}$ Professor Assistente Doutor, FCM UNICAMP; ${ }^{3}$ Fisioterapeuta, Serviço de Fisioterapia do HC UNICAMP; ${ }^{4}$ Doutor em Cirurgia, Departamento de Cirurgia FCM UNICAMP; ${ }^{5}$ Professor Assistente Doutor, Chefe da UTI do HC, UNICAMP; ${ }^{6}$ Doutor em Cirurgia, Departamento de Neurocirurgia, FCM UNICAMP; ${ }^{7}$ Fisioterapeuta, Mestra, Serviço de Fisioterapia do HC UNICAMP; ${ }^{8}$ Professor Titular, Chefe da Metabologia Cirúrgica da FCM, UNICAMP.
}

Recebido 26 Maio 2004, recebido na forma final 9 Agosto 2004. Aceito 1 Outubro 2004.

Dra. Desanka Dragosavac - Rua Olímpio Pattaro 364 Condominio Barão do Café, Barão Geraldo - 13085-045 Campinas SP - Brasil. E-mail: desanka@obelix.unicamp.br 
mitante da PAM. Existem poucos estudos na literatura avaliando a interação da fisioterapia respiratória com a hemodinâmica cerebral no TCE grave.

O objetivo do presente estudo foi avaliar as alterações da PIC, PAM e PPC durante os procedimentos de fisioterapia respiratória em pacientes com TCE grave.

\section{MÉTODO}

O estudo foi prospectivo, intervencionista e aberto, aprovado pelo Comitê de Ética em Pesquisa da UNICAMP ( $N^{\circ} 424 / 2001$ ). Foram colhidos dados de 35 pacientes com TCE grave, sendo 8 do feminino e 27 do masculino, com média de idade de 24,9 anos (variando de 17 a 49 anos).

Os critérios de inclusão foram: - Pacientes com TCE grave (pontuação na Escala de Coma de Glasgow - ECG inicial $\leq 8)$; - PIC $<30 \mathrm{mmHg}$.

Os critérios de exclusão foram: - PIC $\geq 30 \mathrm{mmHg}$; pacientes em morte encefálica.

Foram colhidos os seguintes dados: PAM e PIC; sendo a PPC calculada através da fórmula PPC = PAM - PIC.

Os pacientes foram divididos em três grupos: - Grupo I : PIC inicial 0 - $10 \mathrm{mmHg}$ (10 pacientes); - Grupo II : PIC inicial 11 - $20 \mathrm{mmHg}$ (15 pacientes); - Grupo III: PIC inicial entre $21-30 \mathrm{mmHg}$ (10 pacientes).

A monitorização da PIC foi feita através do cateter subaracnóide². A PAM foi monitorizada através de esfigmomanômetro (monitorização da pressão não invasiva do aparelho multiparamétrico de monitorização hemodinãmica da Philips).

O protocolo de fisioterapia respiratória constituiuse de oito manobras:

pressão manual expiratória com vibração manual costal (M1), pressão manual expiratória com vibração manual diafragmática (M2), drenagem postural associada com tapotagem (M3), pressão manual expiratória com descompressão costal (M4), pressão manual expiratória com descompressão diafragmática (M5), respiração contrariada (M6), respiração localizada (M7) e aspiração endotraqueal (M8).

As medidas foram feitas um minuto após cada manobra, um (M9) e 30 minutos (M 10) depois da última manobra.

A análise estatística foi feita através da análise de variância (ANOVA) e teste de contraste de perfil. O nível de significância adotado foi $5 \%$.

\section{RESULTADOS}

No grupo 1 ( $\mathrm{PIC} \leq 10$ ) houve aumento importante de PIC após a aspiração traqueal (de 6,90 22,33 para $11,40 \pm 4,55 \mathrm{mmHg}, p=0,016)$, manobra de pressão manual expiratória com vibração manual dia-

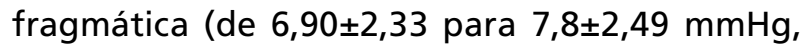
$\mathrm{p}=0,015$ ) e drenagem postural com tapotagem (de $6,90 \pm 2,33$ para $9,20 \pm 2,57 \mathrm{mmHg}, p=0,02)$. Essa mu- dança não foi acompanhada com aumento de PAM, e a PPC, apesar de pequena queda se manteve dentro dos valores normais (Tabela 1).

No grupo 2 (PIC $11-20 \mathrm{mmHg}$ ) houve aumento significativo de PIC após manobra de aspiração traqueal (de 13,67, \pm 20 para $18,73 \pm 6,30 \mathrm{mmHg}$, $\mathrm{p}=0,006)$, com normalização 1 minuto $(16,13 \pm 5,46$ $\mathrm{mmHg}, \mathrm{p}=0,003)$ e 30 minutos depois $(12,73+3,94$ $\mathrm{mmHg}, \mathrm{p}=0,007)$. Essas mudanças não foram acompanhadas de aumentos de PAM e a PPC mantevese dentro da limite da normalidade (Tabela 2). A PPC apresentou melhora após 30 minutos das manobras de fisioterapia respiratória, quando comparado com

Tabela 1. Pressão intracraniana (PIC), pressão arterial média (PAM) e pressão de perfusão cerebral (PPC) em pacientes com TCE grave, após as manobras de fisioterapia, no grupo com PIC $0-10 \mathrm{mmHg}\left({ }^{*} p<0,05\right)$.

\begin{tabular}{lccc}
\hline Manobra & $\begin{array}{c}\text { PIC } \\
\text { Média } \pm \text { DP }\end{array}$ & $\begin{array}{c}\text { PAM } \mathrm{mmHg} \\
\text { Média } \pm \mathrm{DP}\end{array}$ & $\begin{array}{c}\text { PPC } \mathrm{mmHg} \\
\text { Média } \pm \mathrm{DP}\end{array}$ \\
\hline 0 & $6,90 \pm 2,33$ & $97,60 \pm 16,87$ & $90,70 \pm 16,49$ \\
M1 & $7,30 \pm 2,58$ & $97,90 \pm 15,92$ & $90,60 \pm 15,01$ \\
M2 & $7,80 \pm 2,49 *$ & $97,30 \pm 15,91$ & $89,50 \pm 15,33$ \\
M3 & $9,20 \pm 2,57 *$ & $94,30 \pm 12,31$ & $85,10 \pm 11,69$ \\
M4 & $8,60 \pm 2,50$ & $96,30 \pm 17,24$ & $87,70 \pm 16,77$ \\
M5 & $7,80 \pm 2,39$ & $98,40 \pm 18,97$ & $90,60 \pm 18,15$ \\
M6 & $8,00 \pm 2,67$ & $96,20 \pm 20,30$ & $88,20 \pm 20,13$ \\
M7 & $8,40 \pm 2,72$ & $97,90 \pm 20,28$ & $89,50 \pm 19,85$ \\
M8 & $11,40 \pm 4,55 *$ & $102,80 \pm 24,33$ & $91,40 \pm 23,19$ \\
M9 & $9,70 \pm 3,92$ & $103,10 \pm 23,66$ & $93,40 \pm 22.26$ \\
M10 & $7,20 \pm 2,90$ & $98,40 \pm 19,20$ & $91,20 \pm 18,76$ \\
\hline 0, ante
\end{tabular}

0 , antes da fisioterapia; M1-M8, manobras de fisioterapia; M9, um minuto pós-fisioterapia; M10, 30 minutos pós-fisioterapia.

Tabela 2. Pressão intracraniana (PIC), pressão arterial média (PAM) e pressão de perfusão cerebral (PPC) em pacientes com TCE grave, após as manobras de fisioterapia, no grupo com PIC $11-20 \mathrm{mmHg}\left({ }^{*} p<0,05\right)$.

\begin{tabular}{lccc}
\hline Manobra & $\begin{array}{c}\text { PIC } \mathrm{mmHg} \\
\text { Média } \pm \mathrm{DP}\end{array}$ & $\begin{array}{c}\text { PAM } \mathrm{mmHg} \\
\text { Média } \pm \mathrm{DP}\end{array}$ & $\begin{array}{c}\text { PPC } \mathrm{mmHg} \\
\text { Média } \pm \mathrm{DP}\end{array}$ \\
\hline 0 & $13,67 \pm 3,20$ & $99,80 \pm 10,14$ & $86,80 \pm 12,21$ \\
M1 & $13,47 \pm 3,27$ & $100,00 \pm 10,03$ & $86,53 \pm 11,49$ \\
M2 & $13,60 \pm 3,66$ & $100,07 \pm 11,49$ & $86,47 \pm 13,83$ \\
M3 & $14,47 \pm 3,98$ & $98,07 \pm 9,51$ & $83,60 \pm 11,62$ \\
M4 & $14,73 \pm 4,80$ & $99,33 \pm 8,86$ & $84,60 \pm 11,46$ \\
M5 & $15,07 \pm 5,06$ & $98,60 \pm 9,34$ & $84,20 \pm 12,27$ \\
M6 & $14,53 \pm 5,33$ & $97,47 \pm 9,58$ & $82,27 \pm 13,72$ \\
M7 & $14,47 \pm 5,83$ & $97,93 \pm 9,52$ & $84,13 \pm 2,52$ \\
M8 & $18,73 \pm 6,30 *$ & $101,13 \pm 10,55$ & $82,40 \pm 13,27$ \\
M9 & $16,13 \pm 5,46 *$ & $100,73 \pm 10,67$ & $84,60 \pm 12,60$ * \\
M10 & $12,73 \pm 3,94 *$ & $99,47 \pm 8,91$ & $87,13 \pm 10,32$ \\
\hline
\end{tabular}

0 , antes da fisioterapia; M1- M8, manobras de fisioterapia; M9, um minuto pós-fisioterapia; M10, 30 minutos pós-fisioterapia. 
Tabela 3. Pressão intracraniana (PIC), pressão arterial média (PAM) e pressão de perfusão cerebral (PPC) em pacientes com TCE grave, após as manobras de fisioterapia, no grupo com PIC $>21 \mathrm{mmHg}(* p<0,05)$.

\begin{tabular}{lccc}
\hline Manobra & $\begin{array}{c}\text { PIC mmHg } \\
\text { Média } \pm \text { DP }\end{array}$ & $\begin{array}{c}\text { PAM mmHg } \\
\text { Média } \pm \text { DP }\end{array}$ & $\begin{array}{c}\text { PPC mmHg } \\
\text { Média } \pm \text { DP }\end{array}$ \\
\hline 0 & $24,70 \pm 4,47$ & $93,30 \pm 16,75$ & $68,60 \pm 18,51$ \\
M1 & $23,40 \pm 6,02$ & $94,90 \pm 16,22$ & $71,50 \pm 16,49$ \\
M2 & $23,00 \pm 6,25$ & $96,30 \pm 16,04$ & $73,30 \pm 15,96$ \\
M3 & $25,40 \pm 7,01$ & $96,10 \pm 15,50$ & $70.70 \pm 17,59$ \\
M4 & $24,80 \pm 5,22$ & $94,90 \pm 15,21$ & $70,10 \pm 18,16$ \\
M5 & $25,00 \pm 5,06$ & $94,20 \pm 16,98$ & $68,20 \pm 18,49$ \\
M6 & $24,00 \pm 5,35$ & $92,10 \pm 16,89$ & $68,10 \pm 19,78$ \\
M7 & $24,30 \pm 5,38$ & $94,20 \pm 15,24$ & $69.90 \pm 17,89$ \\
M8 & $28,40 \pm 5,42 *$ & $98,10 \pm 15,69$ & $69,70 \pm 18,46$ \\
M9 & $26,60 \pm 4,77$ & $98.80 \pm 15,78$ & $69,20 \pm 17,32$ \\
M10 & $23,10 \pm 3,87 *$ & $95,50 \pm 15,69$ & $72,40 \pm 18,06$ \\
\hline 0, antes
\end{tabular}

0 , antes da fisioterapia; M1 - M8 manobras de fisioterapia; M9 um minuto pós-fisioterapia; M10, 30 minutos pós-fisioterapia.

valores iniciais $(86,80+12,21$ e $87,13+10,32 \mathrm{mmHg}$, retrospectivamente).

No grupo 3 (PIC 21-30 mm Hg) a PIC aumentou após manobra de aspiração traqueal (de 24,70 $\pm 4,47$ para $28,40 \pm 5,42, p=0,0007$ ), sem alteração de PAM e PPC, com normalização 30 minutos depois da manobra $(23,10 \pm 3,87 \mathrm{mmHg}, \mathrm{p}=0,0081)$ (Tabela 3$)$.

\section{DISCUSSÃO}

As manobras de fisioterapia respiratória aplicadas sobre a caixa torácica dos pacientes aumentam a pressão intratorácica, com queda do retorno venoso para o coração e diminuição da PAM. Comprometem também o retorno venoso cerebral, $\mathrm{O}$ que acarreta aumento da PIC PA. $^{3-6}$ Sabendo que a PPC depende do gradiente de PAM e PIC, e que com as manobras fisioterápicas pode ocorrer simultaneamente a queda da PAM e aumento da PIC, interessou-nos saber se essas alterações poderiam comprometer a PPC de modo clinicamente significativo.

Em todos os pacientes houve aumento de PIC, sem aumento de PAM, após a aspiração, porém sempre com manutenção de PPC acima de $60 \mathrm{~mm} \mathrm{Hg}$, voltando aos valores basais um e 30 minutos depois.

A pressão intratorácica (PIT) relaciona-se diretamente com a pressão alveolar, que é notadamente alterada pelas compressões torácicas durante as manobras fisioterápicas.

O aumento da PIT poderia ser prejudicial para o retorno venoso cerebral, pois leva a um incremento da pressão no ventrículo direito e grandes vasos, incluindo a veia cava superior ${ }^{7,8}$. As manipulações do paciente, como a mudança de decúbito, massoterapia de alívio, banho e curativos, podem causar o aumento da PIC durante ${ }^{9-14}$.

A PIT gerada pela tosse e pela manobra de Valsalva determina repercussões no sistema cardiovascular e sistema venoso central, que podem ser comparadas às geradas pelo aumento da PIT determinado pela instalação da PEEP (positive end expira tory pressure). Acredita-se que os efeitos são parecidos. Vários estudos mostraram que o aumento de PIC nessas situações não compromete de modo significativo a pressão de perfusão cerebral ${ }^{4,6,7}$.

Bruya, no momento da aspiração endotraqueal, observou aumento significativo da PIC, devido à tosse. Após período de repouso, os valores da PIC retornaram à normalidade, fato também encontrado em nosso estudo ${ }^{12}$. No presente trabalho, 30 minutos após o final da fisioterapia encontrou-se redução da PIC de 1 a $2 \mathrm{mmHg}(p=0,0072)$ quando comparado com valores iniciais, provavelmente por melhora de ventilação pulmonar. Durante a aspiração endotraqueal há aumento de PIC devido ao reflexo de tosse, porém vários trabalhos mostraram aumento de $\mathrm{PaCO}_{2}$ que, por sua vez, pode piorar a PIC por vasodilatação cerebral. A queda da $\mathrm{PaCO}_{2}$ pós a fisioterapia respiratória pode determinar otimização do tônus vascular e, assim, a PPC ${ }^{12,14}$.

O aumento da PIC pode vir acompanhando de aumento da PAM, principalmente em situações em que a auto-regulação cerebral encontra-se limítrofe ou ausente.

Higiene brônquica, realizada através da instilação endotraqueal de solução salina e ventilação forçada com AMBU, junto com a aspiração endotraqueal, causa aumentos passageiros da PIC.Este aumento não causa conseqüências deletérias para a PPC quando o equilíbrio entre a PAM e a PIC mantem-se íntegro ${ }^{13-16}$. Exitem poucos trabalhos na literatura que avaliam esse assunto, porém todos acharam que a fisioterapia causa pequeno e temporário aumento da PIC, sem alteração de PPC. Portanto, as manobras de fisioterapia podem ser aplicadas em pacientes com TCE com segurança ${ }^{15-18}$.

Como a aspiração endotraqueal causa a tosse e manobra de Valsalva, aumentando significativamente a PIC, na nossa opinião e dos outros autores deve ser feita com muito cuidado, por tempo curto e somente quando necessário ${ }^{17-18}$.

Em conclusão, a aspiração endotraqueal alterou a PIC em todos os grupos, com recuperação rápida um minuto após o término do procedimento. As ma- 
nobras fisioterápicas não afetaram de modo clinicamente significativo a PPC em pacientes com TCE grave, com valores da PIC até $30 \mathrm{mmHg}$. Dessa forma, manobras de fisioterapia respiratória podem ser usadas com segurança em pacientes portadores de TCE grave, com PIC menor que $30 \mathrm{mmHg}$. Certo cuidado deve ser tomado durante a aspiração endotraqueal, sendo ela feita por tempo curto e somente quando necessário.

\section{REFERÊNCIAS}

1. Colli BO. Hipertensão intracraniana: fisiopatologia, diagnóstico e tratamento. J Bras Neurocir 1990;2:25-34.

2. Falcão ALE, Dantas VP Filho, Saraiva JFK, Sardinha LAC, Araújo S, Terzi RGG. Bedside ICP monitoring in head trauma patient by using a simple, safe and low cost methodology. Clin Intens Care 1992;(Suppl)3:112.

3. Bloomfield GL, Ridings PC, Blocher CR, Marmarou A, Surgerman HJ.A proposed relationship between increase intra-abdominal, intrathoracic, and intracranial pressure.Crit Care Med 1997;25:496-503.

4. Fessler H, Brower RG, Wise RA, Permutt S. Efects of positive end-expiratory pressure on the gradient for venous return. Am Rev Respir Dis 1991;143:19-24.

5. Fisher D, Frewen T, Swedlow D. Increase in intracranial pressure during suctioning stimulation versus rise in $\mathrm{PaCO} 2$. Anesthesiology 1982;57:416-417.

6. Georgiadis D, Schwart S, Baumgartner RW, Veltkamp R, Schwab S. Influence of positiveend-expiratory pressureon intracranial press u reand cerebral perfusion pressure in patients with acute stroke. Stroke 2001; 32:2088-2092.
7. Laws AK, Mcintyre RW. Chest physioterapy: A physiological assessment during intermittent positive press u reventilation in respiratory failure Can Anaesth Soc J 1969;16:487-493.

8. Dragosavac D. Interação cardiopulmonar durante a ventilação mecânica. In Carvalho CRR. (ED) Ventilação mecânica, Volume 1 (Básico). São Paulo: Atheneu 2000, Série Clínicas Brasileiras de medicina intensiva: 5(8).

9. Pinsky MR. Cardiovascular effects of ventilatory support and withdrawal. Anesth Analg 1994;79:567-576.

10. Rudy EB, Turner BS, Baun M, Stone KS, Brucia J. Endotracheal suctioning in adults with head injury. Heart Lung 1991;20:667-674.

11. Brucia J, Rudy E. The effects of suction catheter insertion and tracheal stimulation in adults with severe brain injury. Heart Lung 1996;25:295-303.

12. Bruya M. Planned periods of rest in the intensive care unit: nursing care activities and intracranial pressure. J Neuro s u rgNurs 1981;13:184-194.

13. Mitchell $P$, Mauss N. Relation of patient/nurse activity to intracranial pressure variations: a pilot study. Nurs Res 1978;27: 4-10.

14. Ersson U, Carlson H, Mellströn A, Pontén U, Hedstrand U, ackobson $\mathrm{S}$. Observations on intracranial dynamics during respiratory physiotherapy in inconscious neurosurgical patients. Acta Anaesthesiol Scand 1990;34:99-103.

15. Clemente AJ, Hubach SK. Chest physioterapy by the "bag squeezing method": a guide to technique. Physioterapy 1968;54:355-359.

16. Ersson U, Mellströn A, Pontén U, Carlson H. Observation on intracranial dynamics at endotracheal suction (ES), and bag squeezing (BS) in patient with severe brain trauma. Proc Scand Soc Neurosurg, Acta Neurochir 1988;91:155.

17. Brimioulle S, Moraine JJ, Norrenberg D, Kahn RJ. Effects of positioning and exercise on intracranial pressure in a neurosurgical intensive care unit. Phys Ther 1997;77:1682-1689.

18. Koch SM, Fogarty S, Signorino C, Parmley L, Mehlhorn U. Effect of passive range of motion on intracranial pressure in neuro s u rgical patients. J Crit Care 1996;11:176-179. 